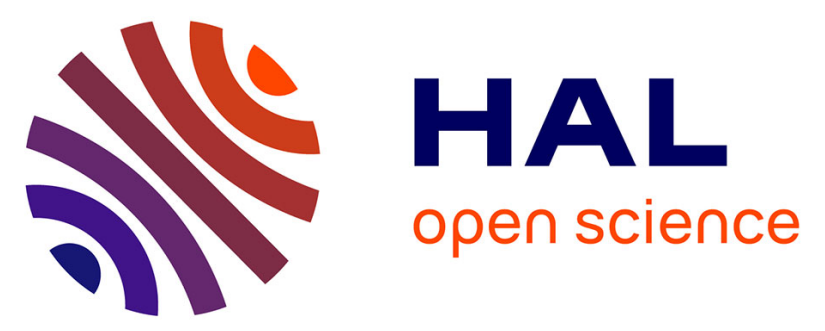

\title{
Imputabilité d'un décès à la méthadone : comparaison de deux méthodes appliquées aux décès associés à une exposition à la méthadone au cours de cinq années d'analyses au CHU de Rennes
}

\author{
C. Hugbart, R. Bouvet, A. Baert, I. Morel, M Le Gueut
}

\section{To cite this version:}

C. Hugbart, R. Bouvet, A. Baert, I. Morel, M Le Gueut. Imputabilité d'un décès à la méthadone : comparaison de deux méthodes appliquées aux décès associés à une exposition à la méthadone au cours de cinq années d'analyses au CHU de Rennes. La Revue de Médecine Légale, 2013, 4 (3), pp.112-120. 10.1016/j.medleg.2013.07.001 . hal-01259444

HAL Id: hal-01259444

https://hal-univ-rennes1.archives-ouvertes.fr/hal-01259444

Submitted on 14 Nov 2018

HAL is a multi-disciplinary open access archive for the deposit and dissemination of scientific research documents, whether they are published or not. The documents may come from teaching and research institutions in France or abroad, or from public or private research centers.
L'archive ouverte pluridisciplinaire HAL, est destinée au dépôt et à la diffusion de documents scientifiques de niveau recherche, publiés ou non, émanant des établissements d'enseignement et de recherche français ou étrangers, des laboratoires publics ou privés. 


\section{Imputabilité d'un décès à la méthadone : comparaison de deux méthodes appliquées aux décès associés à une exposition à la méthadone au cours de cinq années d'analyses au CHU de Rennes}

\section{Methadone-related deaths: Comparison of two methods applied to deaths associated with methadone exposure during five years of analysis at the Rennes university hospital}

C. Hugbart ${ }^{\mathrm{a}}$ R. Bouvet ${ }^{\mathrm{ab}}$ A. Baert ${ }^{\mathrm{a}}$ I. Morel ${ }^{\mathrm{bc}}$ M. Le Gueut ${ }^{\mathrm{ab}}$

a Service de médecine légale, CHU de Rennes, 2, rue Henri-Le-Guilloux, 35033 Rennes cedex 9, France

b Université de Rennes 1, 2, avenue du Professeur-Léon-Bernard, 35043 Rennes cedex, France

c Laboratoire de toxicologie biologique et médicolégale, CHU de Rennes, 2, rue Henri-Le-Guilloux, 35033 Rennes cedex 9, France 
Résumé

But de l'étude : Comparer deux approches de détermination de l'imputabilité d'un décès à une exposition à la méthadone.

Matériel et méthodes: Etude rétrospective (2007-2011) des expertises judiciaires du laboratoire de toxicologie du CHU de Rennes ayant mis en évidence la présence de méthadone dans le sang ou les urines de sujets décédés. L'imputabilité du décès à la méthadone a été discutée selon deux méthodes: la première, dite «classique »était uniquement basée sur la concentration sanguine post mortem de méthadone. La seconde, dite «globale », basée sur l'analyse de facteurs de risques, reposait sur un examen critique de l’ensemble des données recueillies lors des investigations médico-légales.

Résultats : 25 dossiers ont été inclus. Le pourcentage des dossiers sans conclusion était de 56 \% pour la méthode classique contre $24 \%$ pour la méthode globale. Cette dernière approche a permis d'imputer avec certitude le décès à la méthadone dans quatre cas, contre un seul pour la méthode classique. Un xénobiotique substrat ou inhibiteur des cytochromes P450 participant au métabolisme de la méthadone était présent dans $20 \%$ des cas. La présence concomitante de xénobiotiques dépresseurs respiratoires était fréquente (72\%). Le facteur de risque cardiaque le plus fréquent était la présence de xénobiotiques allongeant l'intervalle QT $(36 \%)$.

Conclusion : La méthode fondée sur la seule concentration sanguine en méthadone n'a pas permis de conclure dans plus de $75 \%$ des dossiers étudiés. Une méthode basée sur une approche médico-légale des facteurs de risque s'est montrée plus performante.

Mots clés : méthadone, décès, imputabilité, overdose, intoxication 
Abstract

Purpose of the study : Comparing two approaches for discussing the imputability of methadone-related death.

Methods : A retrospective study was conducted over the period from January 2003 to October 2011, on the forensic toxicology laboratory in Rennes. The records of dead individuals, where the presence of methadone in blood or urine has been established, were selected. The imputability of methadone-related deaths has been established by two approaches: the first one, called "traditionnal" method and was only based on post-mortem blood concentration of methadone. The second, called "global", was based on a critical study of all the data collected during forensic investigations.

Results : 25 cases were included. The average age was 31.4 years. The percentage of cases without conclusion was $56 \%$ for the traditional method against $24 \%$ for the global one. Four deaths have been attributed to methadone for the latter, against just one for the former. Xenobiotic cytochrome P450 substrate or inhibitor was present in $20 \%$ of cases. The concomitant presence of respiratory depressant xenobiotics was common ( $72 \%$ of cases). The most frequently found cardiac risk factors concerned the presence of xenobiotics that prolonged the QT interval (36\% of cases).

Conclusion : The method based on single blood concentration of methadone did not conclude in more than $75 \%$ of the cases studied. A method based on a forensic approach of risk factors proved to be more efficient.

Key words: methadone, death, imputability, overdose, poisoning 
Introduction

En France, l'unique indication de prescription de méthadone est la pharmacodépendance majeure aux opiacés $[1,2]$. Le nombre de patients bénéficiant de ce traitement est en constante augmentation et était estimé à 37700 en 2009 [3]. Une augmentation des décès impliquant la méthadone a été observée en France [4], tout comme aux Etats-Unis [5, 6].

La méthadone est un agoniste des récepteurs opioïdes. Ses propriétés pharmacologiques et sa toxicité sont connues [7-9]. Le métabolisme s'effectue principalement au niveau hépatique où la méthadone subit une $\mathrm{N}$-déméthylation et une cyclisation sans conjugaison via le système des cytochromes P450. Les principales enzymes impliquées dans ce métabolisme sont les cytochromes (CYP) 3A4, 2D6 et 2B6 [10]. La consommation concomitante de xénobiotiques substrats ou inhibiteurs de ces CYP modifie le métabolisme de la méthadone, et peut entrainer une augmentation de sa toxicité. Le métabolite principal est la 2-éthylidène-1,5-diméthyl-3,3diphényl pyrrolidine (EDDP) qui est inactif. La méthadone est principalement éliminée dans les urines, mais aussi dans les selles, la salive, la sueur et les cheveux [11].

Les mécanismes d'un décès par exposition à la méthadone ont été identifiés et peuvent relever soit d'une défaillance respiratoire [11-14] comme pour tout opioïde, soit d'une défaillance cardiaque. Un des effets indésirables de la méthadone est d'allonger l'intervalle QT [13, 15, 16], entrainant un risque de passage en torsade de pointe. Cependant, les cas de torsade de pointe chez des sujets vivants sous méthadone n’ont été principalement observés qu'en présence d'au moins un autre facteur allongeant le QT : hypokaliémie, médicaments allongeant le QT, xénobiotiques cardiotoxiques, xénobiotiques inhibant les CYP métabolisant 
la méthadone, prédisposition génétique responsable d'un syndrome du QT long [17], fortes doses de méthadone... [18-21].

Le médecin légiste doit se prononcer sur l'imputabilité du décès à la méthadone sans avoir, par définition, observé les troubles caractéristiques de l'intoxication. Pour ce faire, il est habituel de tenir compte de la concentration de la substance dans le sang. La pertinence de cette approche est d'ores et déjà considérée comme discutable d'un point de vue médicolégal. En effet, le raisonnement diagnostique doit prendre en compte le sujet dans son ensemble [22] : symptomatologie au moment du décès, circonstances de découverte du corps, existence d'une éventuelle pathologie associée [23], ensemble des substance(s) consommée(s), tolérance à la substance [24, 25].

L'objectif de notre étude est de comparer deux approches de détermination de l'imputabilité d'un décès à une exposition à la méthadone : la méthode dite «classique » uniquement basée sur la concentration sanguine post mortem de méthadone, et une méthode que nous qualifierons de «globale», basée sur l'étude des facteurs de risque, qui examine de manière critique l'ensemble des données recueillies lors des investigations médico-légales et tente de s'affranchir des concentration sanguines post mortem de méthadone.

\section{Matériel et Méthodes}

Il s'agit d'une étude rétrospective, menée sur la période de janvier 2007 à octobre 2011. Dans la base de données des expertises judiciaires du laboratoire de toxicologie du Centre Hospitalier Universitaire de Rennes ont été sélectionnés les dossiers des sujets décédés pour lesquels la présence de méthadone dans le sang ou les urines a été établie. 


\section{Techniques analytiques}

Le screening toxicologique sanguin a été réalisé par une technique de chromatographie en phase liquide couplée à un détecteur à barrette de diodes et a été complété d'un dépistage immunoenzymatique sur automate. La recherche et le dosage de molécules psychoactives (benzodiazépines et apparentés, antidépresseurs, phénothiazines, neuroleptiques) et antalgiques (tramadol et dextropropoxyphène) ont été réalisés par chromatographie liquide couplée à la spectrométrie de masse en tandem (LC/MS/MS). Le dosage des 4 familles de stupéfiants (cannabis, cocaïne, opiacés, amphétamines) a été réalisé par chromatographie couplée à une détection par spectrométrie de masse (GC/MS ou LC-MS-MS). Le dosage de l'éthanol dans le sang total a été réalisé par la méthode de référence [26] qui est une chromatographie en phase gazeuse avec détection par ionisation de flamme. Le dosage des produits de substitution aux opiacés (méthadone et EDDP, buprénorphine et norbuprénorphine) a été réalisé par chromatographie liquide couplée à la spectrométrie de masse en tandem (LC/MS/MS). Ce dosage n'a été pratiqué de manière systématique qu'à partir de novembre 2008.

Lorsqu'un prélèvement urinaire était réalisé, un dépistage par une technique immunochimique a été pratiqué, complété par un screening toxicologique par chromatographie liquide haute performance (HPLC) couplée à un détecteur à barrettes de diodes.

Concernant les cheveux, les échantillons ont été analysés par chromatographie liquide haute performance couplée à un spectromètre de masse en tandem (LC/MS/MS).

\section{Méthodes de discussion de l'imputabilité}

L’imputabilité du décès à la méthadone a été exprimée selon quatre items : non responsable ; potentiellement responsable ; responsable ; conclusion impossible. La première méthode, dite 
«classique », est fondée sur la seule concentration sanguine post mortem de méthadone, en référence aux concentrations dites thérapeutiques, toxiques ou létales. Ces concentrations (tableau 1) sont issues de références françaises [27], et internationales émises par des groupements d'experts toxicologues médico-légaux [28]. La responsabilité de la méthadone en fonction de la concentration a été déterminée comme suit :

- Concentration thérapeutique ou infrathérapeutique : non responsable ;

- Concentration létale : responsable ;

- Concentration toxique / concentration toxique ou létale : potentiellement responsable ;

- Concentration thérapeutique ou toxique / concentration thérapeutique ou toxique ou létale : conclusion impossible.

La seconde méthode a été qualifiée de «globale ». Au terme d'une revue de la littérature de la toxicité de la méthadone, basée sur les propriétés pharmacologiques de la molécule, nous avons identifié des facteurs de risque de toxicité. Ils sont de trois ordres: généraux, cardiaques et pulmonaires (tableau 2). Pour chaque dossier, ces facteurs de risque ont été recherchés à partir de l'ensemble des données recueillies lors des investigations médicolégales : levée de corps, autopsie, analyses toxicologiques et examens anatomopathologiques. La levée de corps et l'autopsie ont permis notamment d'éliminer une autre cause de décès. Ces facteurs de risque ont été discutés quant à leur rôle dans la survenue du décès. La tolérance, lorsque qu'elle était connue de manière objective par analyse d'un prélèvement capillaire, a été prise en compte dans chacune des méthodes.

Résultats

Trente deux dossiers ont été identifiés. Il s'agissait de 30 hommes (94\%) et de 2 femmes (6\%). Exceptés 5 sujets dont l'âge n'était pas connu, l'âge moyen était de 31,4 ans [20 - 44 
ans ; médiane : 33]. Sept dossiers n'ont pas été retenus car aucune information de levée de corps, d'autopsie et/ou d'examens d'anatomo-pathologie n'était disponible, ce qui rendait inapplicable la méthode «globale».

\section{Méthode classique}

La concentration sanguine post mortem de méthadone était comprise entre $0 \mathrm{mg} / \mathrm{L}$ (méthadone uniquement retrouvée dans les urines) et $1,4 \mathrm{mg} / \mathrm{L}$ (médiane : 0,35 mg/L). La conclusion a été possible pour 11 dossiers où la méthadone était estimée comme :

- non responsable du décès pour 9 dossiers,

- potentiellement responsable du décès pour 1 dossier,

- responsable du décès pour 1 dossier.

Il demeurait impossible de conclure pour 14 dossiers (56\%) (Tableau 3).

\section{Méthode globale}

\section{A. Investigations réalisées et contexte}

Sur les 25 cas étudiés, une autopsie a été réalisée pour 24 d'entre eux, dont 7 sans levée de corps préalable. Seul un dossier ne comprenait qu'une levée de corps, sans autopsie. Un examen anatomopathologique a été effectué pour 22 cas, avec analyse d'un ou des deux poumons, du cœur en cas d'anomalie macroscopique et/ou d'un poids supérieur a la normale, et de tout autre organe pouvant mettre en évidence un état antérieur ou une cause de décès. Les cheveux ont été prélevés dans 14 cas, mais n’ont été analysés que dans 3 cas.

Dans la moitié des cas (13 cas sur 25$)$, le contexte de survenue du décès était similaire : à la suite d'une soirée entre amis avec consommation d'alcool et/ou d'autres substances psychoactives, le sujet «s'endormait » et était découvert sans vie plusieurs heures après. La 
présence de flacons de méthadone à proximité du corps a été notée dans un quart des cas $(\mathrm{n}=6)$. Une notion de toxicomanie a été rapportée pour 17 cas, sans précision sur les substances habituellement consommées. Un traitement de substitution par méthadone a été supposé pour 5 cas: il a été rapporté par les enquêteurs pour deux d'entre eux, une prescription nominative a été retrouvée pour les 3 autres sur les lieux du décès ou au domicile du sujet. La date d'initiation du traitement substitutif n'était jamais précisée.

\section{B. Constatations autopsiques}

L'autopsie a mis en évidence un syndrome asphyxique dans la totalité des cas. Le poids du cœur était supérieur à la normale dans 7 cas sur 22 (29,2\%). L'autopsie et l'examen anatomopathologique ont permis de mettre en évidence une autre cause de décès dans 3 cas sur 24 $(12,5 \%)$ : un traumatisme cérébral balistique, une intoxication au monoxyde de carbone, un œdème de Quincke. Il existait un état antérieur cardiaque documenté en histologie dans 4 cas sur $17(23,5 \%)$ : ces 4 sujets avaient une coronaropathie, dont 2 avec des lésions évocatrices d'un infarctus ancien. Un état antérieur hépatique à type de stéatose a été relevé à l'autopsie (4,2\%). L'examen anatomo-pathologique des poumons a principalement mis en évidence des lésions œdémateuses (81\% des cas), sans rupture de septa ni lésions hémorragiques qui seraient évocatrices d'une asphyxie mécanique. Le syndrome asphyxique était accompagné de lésions hémorragiques dans $19 \%$ des cas. L’ensemble des données est résumé dans le tableau 4.

\section{Analyses toxicologiques}

Dans un cas, la méthadone n'a été détectée que dans les urines. Le site de prélèvement sanguin n'était jamais précisé. Sauf dans 1 cas, la méthadone était toujours associée à d'autres 
molécules, en moyenne 4 [0-9; médiane = 4] (tableau 5). La méthadone était associée à de l'alcool dans 18 cas, à des benzodiazépines dans 16 cas, et à du cannabis dans 12 cas. L'analyse toxicologique des cheveux a permis de connaitre l'historique des consommations de 3 sujets : un individu était un consommateur régulier de méthadone. Les deux autres sujets ont été considérés comme naïfs. Une prescription nominative récente de méthadone avait pourtant été retrouvée pour un de ces deux individus, ce qui peut indiquer que le sujet n'était pas observant.

\section{Détermination de l'imputabilité}

Concernant les facteurs de risque généraux : il n'a jamais été possible de déterminer la dose supposée ingérée ainsi que le caractère anormal des circonstances d'ingestion. La tolérance, évaluée uniquement par l'analyse des cheveux afin de s'appuyer sur des données fiables et objectives, n'était connue que pour 3 sujets. Deux d'entre eux n'avait qu'une faible tolérance à la méthadone et étaient donc à risque de présenter des manifestations toxiques voire létales, même pour de faibles doses ingérées. Un xénobiotique substrat ou inhibiteur des CYP était présent dans $20 \%$ des cas (tableau 6). Il s'agissait principalement d'antidépresseurs. Un sujet présentait une stéatose hépatique.

Sur le plan respiratoire, la présence concomitante de xénobiotiques dépresseurs respiratoires était fréquente ( $72 \%$ des cas). Les molécules les plus fréquemment retrouvées étaient les benzodiazépines (52\%), l'alcool (44\%), puis les opiacés (12\%). Deux sujets sur 25 présentaient un état antérieur : l'un avait un emphysème pulmonaire et une stéatose hépatique, l'autre présentait une bronchopneumonie. 
Concernant les facteurs de risque cardiaque, le facteur de risque le plus fréquemment retrouvé concernait la présence de xénobiotiques allongeant l'intervalle QT (36\% des cas), principalement des antidépresseurs et des neuroleptiques. La cocaïne ou son métabolite la benzoylecgonine était retrouvée chez $20 \%$ des individus. Quatre sujets (23,5\%) avaient un état antérieur cardiaque, à type de coronaropathie. Il n'a jamais été retrouvé de traitement hypokaliémiant ; on ne disposait d'aucune information relative à la concentration sanguine de potassium au moment du décès. Il n'a pas été réalisé d'examen génétique à la recherche d'un syndrome congénital du QT long, et aucun antécédent familial, notamment de mort subite, n’a été rapporté.

En raison de la mise en évidence d'une autre étiologie, la responsabilité de la méthadone a pu être exclue dans 4 cas (16\%). La méthadone a été jugée potentiellement responsable dans 11 cas $(44 \%)$, et responsable du décès dans 4 cas $(16 \%)$. Les données n'ont pas permis de conclure dans 6 cas $(24 \%)$.

\section{Comparaison des méthodes}

Le pourcentage des dossiers sans conclusion était de $56 \%$ pour la méthode dite classique contre $24 \%$ pour la méthode globale (tableau 3). Cette dernière approche a permis d'imputer le décès à la méthadone dans 4 cas, contre un seul pour la méthode classique. Parmi les 9 cas où la méthode classique a conclu non responsable, les conclusions issues de l'approche globale ont divergé dans 6 cas où la méthadone a été jugée potentiellement responsable du décès. Pour les 14 cas où la conclusion a été jugée impossible quand la méthode classique était utilisée, l'application de la méthode globale a permis d'apporter une réponse dans 9 cas : la méthadone a été considérée comme non responsable dans 2 cas, potentiellement responsable dans 5 cas et responsable dans 2 cas. 


\section{Discussion}

Notre série de décès associés à une exposition à la méthadone est, de par son effectif et ses caractéristiques démographiques, comparable à d'autres travaux [29, 30, 31]. Bien que de taille limitée, elle a permis d'identifier des similitudes dans le contexte de survenue du décès : le sujet était un homme jeune (environ 30 ans), qui consommait de l'alcool et/ou des substances psychoactives puis s'endormait. Il était découvert décédé plusieurs heures après, généralement par des proches. Un antécédent de toxicomanie et de poly consommation de psychotropes était souvent rapporté. La méthadone était fréquemment associée à d'autres xénobiotiques dans le sang (en moyenne 4 molécules), principalement de l'alcool, des benzodiazépines et du cannabis.

L'application de la méthode basée sur la seule concentration sanguine post mortem de méthadone pour déterminer le rôle de la molécule dans la survenue du décès a confirmé la faible performance de cette approche. La part de dossiers sans conclusion était de $56 \%$, alors que la responsabilité de la méthadone n'a pu être affirmée que dans un cas. Cette faible performance peut s'expliquer notamment par l'absence de consensus entre les référentiels et par le chevauchement des plages de concentrations considérées comme thérapeutiques, toxiques et létales, ce chevauchement conduisant, pour une même concentration, à des conclusions divergentes. La responsabilité de la méthadone a été exclue dans 9 cas, où la concentration était inférieure à $0,2 \mathrm{mg} / \mathrm{L}$ (valeur thérapeutique). La méthode globale a estimé, pour ces 9 dossiers, que la méthadone était potentiellement responsable dans 5 cas. 
Il est admis qu'une concentration supérieure à $0.2 \mathrm{mg} / \mathrm{L}$ est suffisante pour provoquer la mort par dépression respiratoire. Cependant, des concentrations supérieures à $1 \mathrm{mg} / \mathrm{L}$, considérées par certains auteurs comme létales [11], ont été mesurées chez des sujets sous traitement de substitution. A l'inverse, il existe également un risque de sous diagnostiquer une intoxication létale. Parmi les décès mettant en évidence une concentration sanguine très basse, a priori non toxique, la méthadone peut tout de même être à l'origine de la mort : elle peut par exemple provoquer une longue période d'inconscience menant fréquemment à une inhalation. La molécule est dans ce cas métabolisée durant cette période d'inconscience, expliquant la faible concentration retrouvée, alors même qu'elle est la cause du décès [32, 33]. De même, une association entre méthadone et mort subite par trouble du rythme cardiaque a été démontrée, même à des concentrations considérées comme thérapeutiques [13]. L'intérêt pour le médecin légiste de recourir à ces valeurs dites de « référence » apparait donc limité.

S'il existe une relation incontestable entre la dose absorbée et l'effet de la molécule sur l'organisme, la concentration sanguine de méthadone n'est le reflet ni de la dose ingérée ni de l'effet de la molécule, en raison d'une variation de sa pharmacocinétique [34] (biodisponibilité notamment) et des mécanismes de tolérance développés par le sujet [35-38]. Ce phénomène de tolérance est à l'origine d'un chevauchement des plages de concentration dites thérapeutique, toxique et létale, et rend d'autant plus délicate l'interprétation des analyses toxicologiques : une concentration sanguine considérée comme thérapeutique pour un sujet tolérant, c'est-à-dire consommateur régulier d'opioïdes, pourra être toxique voire létale pour un sujet naïf. On estime que le risque de dépression respiratoire fatale est supérieur lors de l'initiation du traitement, notamment lors des 2 premières semaines [24, 36]. Cette absence de corrélation entre concentration sanguine et effet de la molécule permet d'affirmer que l'établissement d'un lien de causalité entre exposition à la méthadone et survenue du 
décès ne peut s'appuyer sur la seule concentration sanguine. Cette approche doit d'autant plus être évitée en post mortem que les concentrations obtenues à partir des prélèvements réalisés sur le cadavre ne sont pas le reflet de la concentration sanguine au moment du décès, en raison des variations de concentrations entre les sites de prélèvement, et des phénomènes de redistribution post mortem [32, 39-41]. Ces phénomènes, correspondant aux mouvements d'une molécule dans l'organisme, se traduisent par une concentration sanguine significativement plus élevée à distance qu'au moment du décès, et représentent le principal facteur affectant l'interprétation de la concentration post mortem d'un xénobiotique. Le principal mécanisme impliqué est la diffusion et le relargage de la molécule après la mort, à partir des tissus et organes qui la contiennent en haute concentration (habituellement le foie et les poumons), vers les vaisseaux sanguins cardiaques et pulmonaires situés à proximité. La méthadone est particulièrement affectée par ces phénomènes. Les concentrations sanguines post mortem sont ainsi plus élevées dans le sang cardiaque (jusqu'à trois fois supérieures) que dans le sang périphérique $[25,42,43]$. La concentration sanguine post mortem de méthadone représente donc un élément d'orientation et non de certitude. Pour autant, la concentration sanguine de méthadone nécessite toujours d'être mesurée. Les résultats doivent davantage être examinés de manière semi quantitative, c'est pourquoi les références de concentration ne sont pas inutiles.

L'application de la méthode globale a permis d'imputer le décès à la méthadone dans 4 cas contre un seul pour la méthode classique. La méthadone n'a été exclue que dans les situations où une autre cause de décès a été mise en évidence, limitant ainsi le risque de sous évaluation. Parmi les 14 cas où il était impossible de conclure par la méthode classique, il a été possible d'apporter une réponse pour 9 cas. Mais les interprétations formelles (retenant ou excluant la responsabilité de la méthadone), et donc exploitables par les autorités judiciaires, n'ont 
concerné qu'une proportion restreinte de dossiers (32\%). Ces résultats semblent cependant pouvoir être améliorés notamment par le perfectionnement des pratiques.

Ainsi, les levées de corps doivent être systématiques, afin de recueillir des données objectives, d'orienter les enquêteurs sur les éléments importants à rechercher : antécédent familial de mort subite, traitement personnel, stupéfiants consommés, existence d'un suivi dans un centre d'addictologie avec si possible mise à disposition du dossier médical, date d'initiation de l'éventuel traitement substitutif... L'autopsie doit toujours être réalisée. Elle recherche un syndrome asphyxique, un état antérieur et une autre cause de décès. Le prélèvement sanguin est indispensable et doit être effectué préférentiellement dans la veine fémorale [40, 42, 44]. Les cheveux, ou à défaut les poils pubiens, doivent être prélevés et analysés. Cette analyse est indispensable pour confirmer de manière objective les informations rapportées par les enquêteurs sur les habitudes de consommations du sujet et déterminer sa tolérance à la méthadone [45, 46]. Dans notre série, la connaissance de l'état de tolérance du sujet aurait pu permettre, pour les cas où la méthadone semble responsable du décès $(24 \%)$, d'affirmer son imputabilité ou au contraire de minimiser son rôle. Nous n'avons pas été en mesure d'apporter de conclusion pour 3 cas, en raison de l'absence d'analyse des cheveux, pourtant prélevés. Le médecin légiste doit s'attacher à exposer au magistrat la nécessité de l'exploitation de ces prélèvements. Pour discuter l'imputabilité d'un décès à la méthadone, une réflexion collégiale entre médecins légistes, toxicologues analystes et anatomo-pathologistes, permettant une synthèse de l'ensemble des informations recueillies, semble indispensable. Un guide d'aide à l'interprétation des explorations médicojudiciaires, adapté aux décès en lien avec une exposition à la méthadone, a été proposé sous forme d'algorithme (figure 1). 
Des analyses à visée génétique pourraient être envisagées. Les variations interindividuelles de la biodisponibilité et de la demi-vie de la méthadone s'expliquent en partie par une variabilité pharmacogénétique qui concerne les gènes codant les enzymes impliqués dans son métabolisme, et principalement les enzymes du système cytochrome P450 CYP2D6 et CYP2B6. La méthadone est administrée sous forme de racémique et l'énantiomère $\mathrm{R}$ est responsable de la plupart de l'activité pharmacologique. Six pour cent de la population caucasienne présente un génotype particulier de l'enzyme CYP 2B6, dont le substrat est essentiellement l'isomère S-méthadone. Ces sujets sont des métaboliseurs lents concernant l'isomère $S$, responsable des effets secondaires cardiaques par allongement du QT, et ont un risque accru de présenter une concentration plasmatique de méthadone, et plus particulièrement de l'isomère $S$, plus élevée $[9,47]$. La mise en évidence de ce génotype constituerait donc un facteur de risque d'intoxication létale à la méthadone $[15,48]$. Sept à huit pour cent de la population caucasienne est déficiente génétiquement en CYP 2D6, ce qui leur conférerait une moindre capacité à métaboliser la méthadone. Il semble cependant exister une discordance entre le génotype et son retentissement phénotypique [49], le polymorphisme génétique du CYP 2D6 n'ayant qu'un impact mineur sur la pharmacocinétique et la pharmacodynamie de la méthadone $[47,50]$, et ne modifiant pas de manière significative les concentrations plasmatiques de méthadone [51].

Enfin, la discussion des cas d'imputabilité d'un décès à la méthadone nous confronte à la recherche des causes génétiques d'un décès de survenue rapide chez un sujet jeune, ce qui pose la question d'une étude génétique cardiaque systématique à la recherche d'un syndrome du QT long congénital, qui est associé à un risque de troubles du rythme ventriculaire graves et potentiellement mortels. Les données actuelles sont en faveur d'une prévalence approximative de 1/5000. Huit gènes modifiés, codant pour des canaux ioniques cardiaques, 
ont été identifiés comme responsables de ce syndrome. Les 3 formes génétiques les plus souvent rencontrées sont KCNQ1 (50\%), KCNH2 (45\%), et SCN5A (4\%) [52]. Il nous semble donc pertinent de prélever systématiquement un échantillon splénique, de le faire placer sous scellé et de le conserver. Si nous avons déjà pu obtenir du magistrat la réalisation d'une telle analyse génétique dans des cas de mort subite chez des sujets sportifs, cela n'a pas encore été le cas - dans notre pratique - en cas d'exposition à la méthadone. Il n'est pas pour l'instant dans la culture des magistrats de requérir ces analyses à visée génétique, or elles sont désormais accessibles à des niveaux de prix acceptables et comparables aux analyses complémentaires de routine médico-légale ; le médecin légiste doit donc s'efforcer d'exposer l'intérêt de l'exploitation de ces prélèvements et d'en convaincre les magistrats.

\section{Conclusion}

Nous avons comparé deux méthodes pour imputer un décès à l'exposition à la méthadone. La méthode fondée sur la seule concentration sanguine en méthadone n'a pas pu conclure dans plus de $75 \%$ des dossiers étudiés. Une méthode que nous qualifions de globale, basée sur une approche médico-légale des facteurs de risque, a semblé plus performante. Si elle n'est pas exempte de limites, celles-ci peuvent être en partie corrigées par l'amélioration des pratiques et l'optimisation des investigations.

\section{Conflit d'intérêts : aucun}




\section{Références}

1. Laqueille $X$, Vazquez V. Aspects pharmacologiques et neurobiologiques de la méthadone dans le traitement des héroïnomanies. Ann Med Psychol 2004; 162: 302306.

2. Lobmaier P, Gossop M, Waal H, Bramness J. The pharmacological treatment of opioid addiction - a clinical perspective. Eur J Clin Pharmacol 2010; 66(6): 537-545.

3. Données nationales - Séries statistiques http://www.ofdt.fr/BDD_len/seristat/00028.xhtml, consulté le 3 avril 2012.

4. Afssaps. Rapports/Synthèses : Stupéfiants et psychotropes - résultats de l'enquête DRAMES. http://www.afssaps.fr/Afssaps-media/Publications/Rapports-SynthesesStupefiants-et-psychotropes, consulté le 3 avril 2012.

5. Products - Health E Stats - Poisoning and Methadone-related Deaths - 1995-2005. http://www.cdc.gov/nchs/data/hestat/poisoning/poisoning.htm, consulté le 3 avril 2012.

6. Bunn TL, Yu L, Spiller HA, Singleton M. Surveillance of methadone-related poisonings in Kentucky using multiple data sources. Pharmacoepidemiol Drug Saf 2010; 19(2): 124-131.

7. Moffat AC, Osselton MD, Widdop B, Watts J Ed. Clarke's analysis of drugs and poisons. 4th ed. London : Pharmaceutical Press, 2008, p. 1648-1650.

8. Eysseric H, Allibe N. Médicaments de substituion aux opiacés. In : Kintz P. Traité de toxicologie médico-judiciaire. $2^{\mathrm{e}}$ éd. Paris : Elsevier-Masson, 2012. p. 426-440.

9. Clark JD. Understanding methadone metabolism. Anesthesiology 2008; 108(3): 351352.

10. Oda Y, Kharasch ED. Metabolism of methadone and levo-alpha-acetylmethadol (LAAM) by human intestinal cytochrome P450 3A4 (CYP3A4): potential contribution of intestinal metabolism to presystemic clearance and bioactivation. J Pharmacol Exp Ther 2001; 298(3): 1021-1032.

11. Corkery JM, Schifano F, Ghodse AH, Oyefeso A. The effects of methadone and its role in fatalities. Hum Psychopharmacol 2004; 19(8): 565-576.

12. Mégarbane B, Buisine A, Jacobs F, Résière D, Chevillard L, Vicaut E, Baud FJ. Prospective comparative assessment of buprenorphine overdose with heroin and methadone: clinical characteristics and response to antidotal treatment. J Subst Abuse Treat 2010; 38(4): 403-407.

13. Chugh SS, Socoteanu C, Reinier K, Waltz J, Jui J, Gunson K. A community-based evaluation of sudden death associated with therapeutic levels of methadone. Am $\mathbf{J}$ Med 2008; 121(1): 66-71. 
14. Mégarbane B, Declèves X, Bloch V, Bardin C, Chast F, Baud FJ. Case report: quantification of methadone-induced respiratory depression using toxicokinetic/toxicodynamic relationships. Crit Care 2007; 11(1): R5.

15. Ansermot N, Albayrak O, Schlapfer J, Crettol S, Croquette-Krokar M, Bourquin M, Déglon JJ, Faouzi M, Sherbaum N, Eap CB. Substitution of (R, S)-methadone by (R)methadone: impact on QTc interval. Arch Intern Med 2010; 170(6): 529-536.

16. Lin C, Somberg T, Molnar J, Somberg J. The effects of chiral isolates of methadone on the cardiac potassium channel IKr. Cardiology 2009; 113(1): 59-65.

17. Thanavaro KL, Thanavaro JL. Methadone-induced torsades de pointes: a twist of fate. Heart Lung 2011; 40(5): 448-453.

18. Sticherling C, Schaer BA, Ammann P, Maeder M, Osswald S. Methadone-induced torsade de pointes tachycardias. Swiss Med Wkly 2005; 135(19-20): 282-285.

19. Krantz MJ, Martin J, Stimmel B, Mehta D, Haigney MCP. QTc interval screening in methadone treatment. Ann Intern Med 2009 17; 150(6): 387 -395.

20. Perrin-Terrin A, Pathak A, Lapeyre-Mestre M. QT interval prolongation: prevalence, risk factors and pharmacovigilance data among methadone-treated patients in France. Fundam Clin Pharmacol 2011; 25(4): 503-510.

21. Fredheim OMS, Moksnes K, Borchgrevink PC, Kaasa S, Dale O. Clinical pharmacology of methadone for pain. Acta Anaesthesiol Scand 2008 1; 52(7): 879889.

22. Modesto-Lowe V, Brooks D, Petry N. Methadone deaths: risk factors in pain and addicted populations. J Gen Intern Med 2010; 25 (4): 305-309.

23. Darke S, Duflou J, Torok M. The comparative toxicology and major organ pathology of fatal methadone and heroin toxicity cases. Drug Alcohol Depend 2010 1; 106(1): 16.

24. Cornish R, Macleod J, Strang J, Vickerman P, Hickman M. Risk of death during and after opiate substitution treatment in primary care: prospective observational study in UK General Practice Research Database. Br Med J 2010; 341: c5475.

25. Shields LBE, Hunsaker III JC, Corey TS, Ward MK, Stewart D. Methadone toxicity fatalities: a review of medical examiner cases in a large metropolitan area. J Forensic Sci 2007; 52(6): 1389-1395.

26. Liebig HM, Buelow HJ, Kallmayer R. Quantitation of endogenous aliphatic alcohols in serum and urines. J Chromatogr 1982; 239: 343-349.

27. SFTA website. http://www.sfta.org/commissions/COFRAC168/TOX280clin.pdf, consulté le 21 janvier 2012

28. TIAFT website. http://www.tiaft.org/, consulté le 2 avril 2012. 
29. Pirnay S, Borron S, Giudicelli C, Tourneau J, Baud F, Ricordel I. A critical review of the causes of death among post-mortem toxicological investigations: analysis of 34 buprenorphine-associated and 35 methadone-associated deaths. Addiction 2004; 99(8): 978-988.

30. Ben Khelil M, Tracqui A, Jamey C, Ludes B. Intoxications mortelles aux opiacés/opioïdes expertisées dans le laboratoire de toxicologie médico-légale de l'IML de Strasbourg durant la période 2000-2009. La Revue de Médecine Légale 2012; 3 (1): 19-26.

31. Eiden C, Cathala P, Mathieu-Daude JC, Marson B, Baccino E, Leglise Y, et al. Methadone-related Deaths in Montpellier and Region, from 2000 to 2010. Therapie 2012; 67: 515-522

32. Milroy C, Forrest A. Methadone deaths: a toxicological analysis. J Clin Pathol 2000; 53(4): 277-281.

33. Jones A, Holmgren A, Ahlner J. Blood methadone concentrations in living and deceased persons: variations over time, subject demographics, and relevance of coingested drugs. J Anal Toxicol 2012; 36(1): 12-18.

34. Eap CB, Buclin T, Baumann P, Interindividual variability of the clinical pharmacokinetics of methadone: implications for the treatment of opioid dependence. Clin Pharmacokinet 2002; 41: 1153-1193.

35. Buster MCA, van Brussel GHA, van den Brink W. An increase in overdose mortality during the first 2 weeks after entering or re-entering methadone treatment in Amsterdam. Addiction 2002; 97(8): 993-1001.

36. Karch SB, Stephens BG. Toxicology and pathology of deaths related to methadone: retrospective review. West J Med 2000; 172(1): 11-14.

37. Zador D, Sunjic S. Deaths in methadone maintenance treatment in New South Wales, Australia 1990-1995. Addiction 2000; 95(1): 77-84.

38. Athanasos P, Smith CS, White JM, Somogyi AA, Bochner F, Ling W. Methadone maintenance patients are cross-tolerant to the antinociceptive effects of very high plasma morphine concentrations. Pain 2006; 120(3): 267-275.

39. Jones GR. Posmortem toxicology. In : Clarke's analytical forensic toxicology, edited by Jickells S, Negrusz A. London: Pharmaceutical Press, 2008: 191-217.

40. Forrest A. Obtaining samples at post mortem examination for toxicological and biochemical analyses. J Clin Pathol 1993; 46(4): 292-296.

41. Pounder DJ. The nightmare of postmortem drug changes. Leg Med. 1993; 163-191.

42. Vinet B. La méthadone, indication clinique, pharmacologie, surdosage et mesure dans les liquides biologiques. Ann Biol Clin Qué 2006; 43(3): 47-50.

43. Caplehorn J, Drummer O. Methadone dose and post-mortem blood concentration. Drug Alcohol Rev 2002; 21: 329-333. 
44. Pelissier-Alicot AL, Gaulier JM, Champsaur P, Marquet P. Mécanismes de la redistribution post-mortem des xénobiotiques: le point sur l'état actuel des connaissances. Ann Toxicol Anal 2001; 13(1): 1-17.

45. Chambert S. Apport de l'analyse toxicologique des cheveux dans le diagnostic de décès toxiques impliquant la méthadone [thèse]. Grenoble : université Joseph Fourier; 2009. p. 1-91.

46. Kintz P, Evans J, Villain M, Cirimele V. Interpretation of hair findings in children after methadone poisoning. Forensic Sci Int 2010; 196 (1-3): 51-54.

47. Crettol S, Déglon J-J, Besson J, Croquette-Krokar M, Hämmig R, Gothuey I, et al. ABCB1 and cytochrome P450 genotypes and phenotypes: influence on methadone plasma levels and response to treatment. Clin. Pharmacol. Ther. 2006;80(6):668-681.

48. Wang S-C, Ho I-K, Tsou H-H, Tian J-N, Hsiao C-F, Chen C-H, et al. CYP2B6 Polymorphisms Influence the Plasma Concentration and Clearance of the Methadone S-Enantiomer. J Clin Psychopharmacol. 2011 août;31(4):463-469.

49. Shiran MR, Chowdry J, Rostami-Hodjegan A, Ellis SW, Lennard MS, Iqbal MZ, et al. A discordance between cytochrome P450 2D6 genotype and phenotype in patients undergoing methadone maintenance treatment. Br J Clin Pharmacol. 2003;56(2):220 224.

50. Kupiec TC, Raj V, Vu N. Pharmacogenomics for the forensic toxicologist. J Anal Toxicol. 2006;30(2):65-72.

51. Wong SH, Wagner MA, Jentzen JM, Schur C, Bjerke J, Gock SB, et al. Pharmacogenomics as an aspect of molecular autopsy for forensic pathology/toxicology: does genotyping CYP 2D6 serve as an adjunct for certifying methadone toxicity? J. Forensic Sci. 2003;48(6):1406-1415.

52. Goldenberg I, Moss AJ. Long QT syndrome. J Am Coll Cardiol 2008; 51(24):22912300 


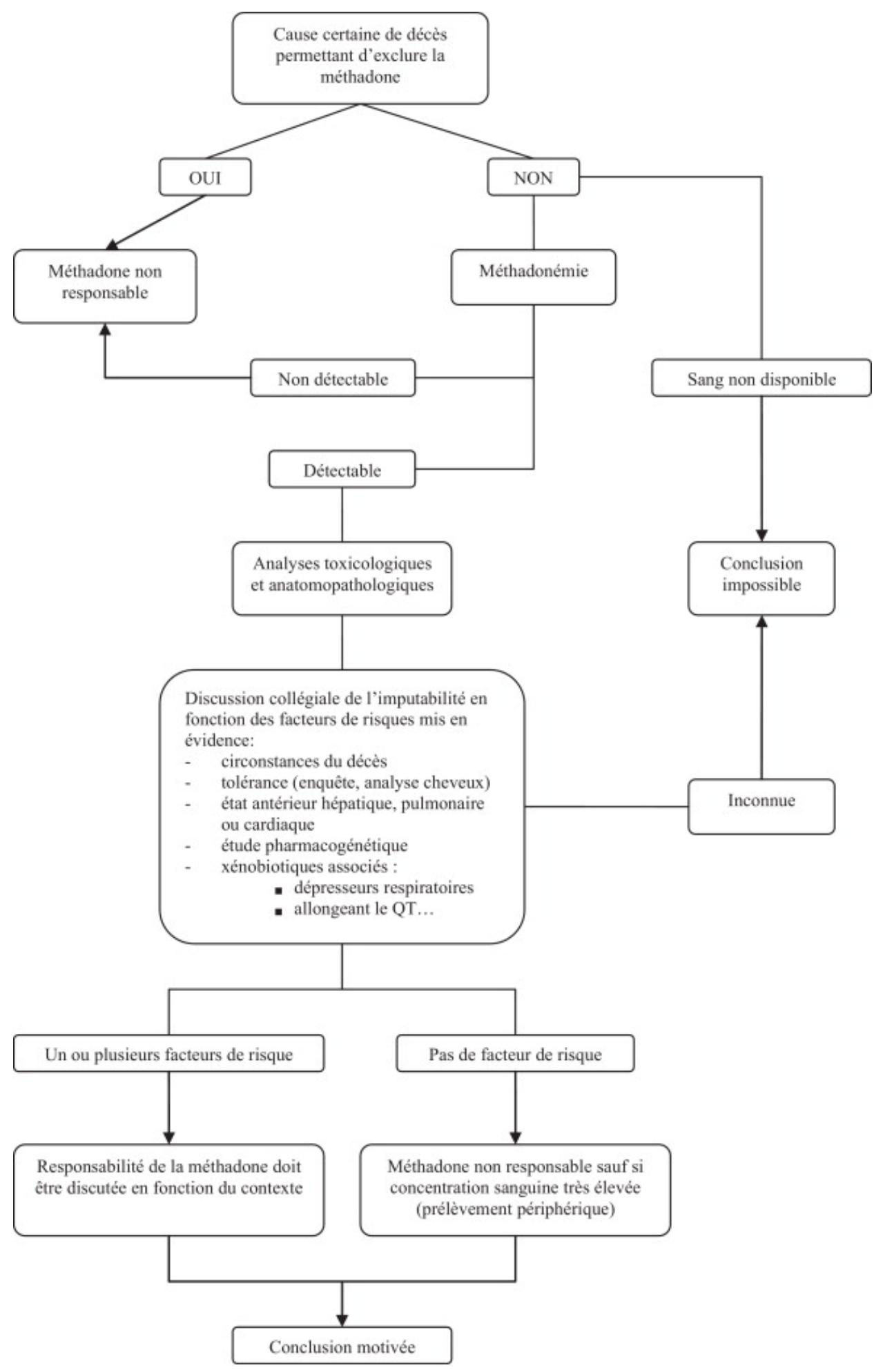


Tableau 1. Concentration thérapeutique, toxique et létale selon The International Association of Forensic Toxicologists (TIAFT) et la Société française de toxicologie analytique (SFTA).

\section{Concentration sanguine}

\begin{tabular}{llll}
\multicolumn{1}{c}{ Thérapeutique $(\mathbf{m g} / \mathbf{L})$} & \multicolumn{1}{c}{ Toxique $(\mathbf{m g} / \mathbf{L})$} & Létale $(\mathbf{m g} / \mathbf{L})$ \\
\hline TIAFT & $0,05-0,5$ & $\begin{array}{l}\text { Naîf : } 0,2 \\
\text { Consommateur : }>0,75\end{array}$ & $0,2-1$ \\
& & & \\
SFTA & $0,05-1$ & $1-2$ & 1,4
\end{tabular}

Tableau 2. Facteurs de risque de toxicité de la méthadone.

\begin{tabular}{ll} 
Facteurs de risque respiratoires & Facteurs de risque cardiaques \\
\hline Pathologie pulmonaire & Pathologie cardiaque \\
Xénobiotiques dépresseurs respiratoires & Xénobiotiques allongeant le QT/cardiotoxiques \\
& Hypokaliémie \\
& Syndrome du QT long
\end{tabular}

Forte dose de méthadone

Faible tolérance

Circonstances inhabituelles de consommation

Pathologie hépatique

Xénobiotiques substrats des CYP 3A4, 2B6 ou 2D6 
Tableau 3. Imputabilité de la méthadone en fonction de la méthode utilisée.

\begin{tabular}{|c|c|c|}
\hline Imputabilité de la méthadone & Méthode classique & Méthode globale \\
\hline \multirow[t]{2}{*}{ Non responsable } & 9 cas & 4 cas \\
\hline & $36 \%$ & $16 \%$ \\
\hline \multirow[t]{2}{*}{ Potentiellement responsable } & 1 cas & 11 cas \\
\hline & $4 \%$ & $44 \%$ \\
\hline \multirow[t]{2}{*}{ Responsable } & 1 cas & 4 cas \\
\hline & $4 \%$ & $16 \%$ \\
\hline \multirow[t]{2}{*}{ Conclusion impossible } & 14 cas & 6 cas \\
\hline & $56 \%$ & $24 \%$ \\
\hline
\end{tabular}


Tableau 4. Constatations autopsiques et anatomopathologiques.

\begin{tabular}{lc} 
Constatations autopsiques & $n(\%)$ \\
\hline Syndrome asphyxique & $n=24$ \\
Poids du cœur supérieur à la normale & $24(100)$ \\
Hépatopathie & $7(29,2)$ \\
Autre cause de décès & $1(4,2)$ \\
\hline
\end{tabular}

Constatations anatomopathologiques

Poumon

$n=22$

Asphyxie œdémateuse

$18(81)$

Asphyxie avec composante hémorragique

$4(19)$

Inhalation

$4(19)$

Pathologie

$2(14,5)$ 
Cœur

Dans les limites de la normale

Athérosclérose/coronaropathie

Hypertrophie ventriculaire gauche

Infarctus ancien

Foie

Stéatose

Aucune lésion

Tractus ORL

Lésions traumatiques

@Edème interstitiel

Aucune lésion $n=17$

$12(70,5)$

$4(23,5)$

$2(11,7)$

$2(11,7)$

$n=1$

$0(0)$

$1(100)$

$n=3$

0 (0)

$1(33,3)$

$2(66,6)$ 
Tableau 5. Autres substances associées à la méthadone dans le sang.

$\begin{array}{lc} & n=24(\%) \\ \text { Alcool } & 18(75) \\ \text { Benzodiazépines } & 16(66,7) \\ \text { Diazépam } & 7(19,2) \\ \text { Oxazépam } & 9(37,5) \\ \text { Nordazépam } & 9(37,5) \\ \text { Témazépam } & 2(8,3) \\ \text { Alprazolam } & 1(4,2) \\ \text { Tétrazépam } & 1(4,2)\end{array}$

Cannabis et dérivés 
Phénothiazine et autres neuroleptiques

$6(25)$

Amisulpride

$1(4,2)$

Loxapine

$1(4,2)$

Cyamémazine

$2(8,3)$

Acéprométazine

$2(8,3)$

Olanzapine

$1(4,2)$

Opiacés autres que la méthadone

$5(20,8)$

6-MAM

$1(4,2)$

Morphine

$1(4,2)$

Codéine

$2(8,3)$

Norcodéine

$2(8,3)$

Glucuronide de morphine

$1(4,2)$

Cocaïne et dérivés

$5(20,8)$ 
Sertraline

Fluvoxamine

Tableau 6. Xénobiotiques substrats ou inhibiteurs des cytochromes, associés à la méthadone dans le sang.

Xénobiotiques subtrats ou inhibiteurs des CYP

Clomipramine

Buprénorphine

Fluvoxamine

Tramadol

Sertraline $n=5(100 \%)$

$1(20)$

$1(20)$

$1(20)$

$1(20)$

$1(20)$ 